PROCEEDINGS OF THE

AMERICAN MATHEMATICAL SOCIETY

Volume 125, Number 9, September 1997, Pages 2599-2604

S 0002-9939(97)03976-2

\title{
GENERALIZED QUASILINEARIZATION METHOD FOR A SECOND ORDER ORDINARY DIFFERENTIAL EQUATION WITH DIRICHLET BOUNDARY CONDITIONS
}

\author{
JUAN J. NIETO
}

(Communicated by Hal L. Smith)

\begin{abstract}
We study the existence and approximation of solutions for a nonlinear second order ordinary differential equation with Dirichlet boundary value conditions. We present a generalized quasilinearization technique to obtain a sequence of approximate solutions converging quadratically to a solution.
\end{abstract}

\section{INTRODUCTION}

The classical method of quasilinearization offers an approach for obtaining approximate solutions to nonlinear equations $[1,2]$. It requires, roughly speaking, that the nonlinearity is convex. Recently, the method of quasilinearization was generalized by not demanding this convexity condition. Indeed, in [5] the authors obtained a sequence of approximate solutions converging quadratically to a solution of an initial value problem for a first order ordinary differential equation. The same result was presented in $[6,7]$ for the periodic boundary value problem for first order ordinary differential equations. In $[9,10]$ a quasilinearization technique for a boundary value problem for a second order ordinary differential equation was developed.

In this paper we study the existence and approximation of solutions for second order ordinary differential equations with Dirichlet boundary value conditions. In section 2, we cite some well known results on the linear Dirichlet problem and then recall the upper and lower solution method and the monotone iterative technique. In section 3 we present our principal result. We develop the method of quasilinearization for a nonlinear Dirichlet problem and obtain a sequence of approximate solutions converging quadratically to a solution of the problem.

Finally, we note that our main result is new since the boundary conditions considered in $[9,10]$ do not include the case of Dirichlet boundary conditions.

Received by the editors March 13, 1996.

1991 Mathematics Subject Classification. Primary 34A45, 34B15.

Key words and phrases. Dirichlet problem, generalized quasilinearization, quadratic convergence.

The author's research was partially supported by D.G.I.C.Y.T. (Spain), project PB94-0610, and by EC Network, CHRX-CT94-0555. 


\section{Preliminary Results}

In the space $C(\Omega)$ we consider the usual uniform norm, that is,

$$
\|u\|=\max \{|u(x)|: x \in \Omega\} .
$$

It is well known that the Dirichlet problem

$$
\begin{gathered}
-u^{\prime \prime}(x)=\lambda u(x), \quad x \in \Omega=[0, \pi], \\
u(0)=u(\pi)=0,
\end{gathered}
$$

has a nontrivial solution if and only if $\lambda=n^{2}$ for some $n=1,2, \ldots$ In consequence, if $\lambda \neq n^{2}, n=1,2 \ldots$, then for any $\sigma \in C(\Omega)$, the problem

$$
\begin{gathered}
-u^{\prime \prime}(x)-\lambda u(x)=\sigma(x), \quad x \in \Omega, \\
u(0)=u(\pi)=0,
\end{gathered}
$$

has a unique solution given by

$$
u(x)=\int_{0}^{\pi} G_{\lambda}(x, y) \sigma(y) d y .
$$

Here, $G_{\lambda}$ is the Green's function given by

$$
G_{\lambda}(x, y)=\frac{1}{\sqrt{-\lambda} \sinh \sqrt{-\lambda} \pi}\left\{\begin{array}{l}
\sinh [\sqrt{-\lambda}(\pi-x)] \sinh [\sqrt{-\lambda} y], 0 \leq y \leq x \leq \pi \\
\sinh [\sqrt{-\lambda} x] \sinh [\sqrt{-\lambda}(\pi-y)], 0 \leq x \leq y \leq \pi
\end{array}\right.
$$

for $\lambda<0$;

$$
G_{0}(x, y)=\frac{1}{\pi} \begin{cases}y(\pi-x), & 0 \leq y \leq x \leq \pi \\ x(\pi-y), & 0 \leq x \leq y \leq \pi\end{cases}
$$

for $\lambda=0 ;$ and

$$
G_{\lambda}(x, y)=\frac{1}{\sqrt{\lambda} \sin \sqrt{\lambda} \pi} \begin{cases}\sin [\sqrt{\lambda}(\pi-x)] \sin [\sqrt{\lambda} y], & 0 \leq y \leq x \leq \pi, \\ \sin [\sqrt{\lambda} x] \sin [\sqrt{\lambda}(\pi-y)], & 0 \leq x \leq y \leq \pi\end{cases}
$$

for $\lambda>0$, and $\lambda \neq n^{2}, n=1,2, \ldots$

From these expressions we see that $G_{\lambda} \geq 0$ for $\lambda<1$. Thus, we have the following comparison result.

Lemma 2.1 (Maximum principle). If $\lambda<1$ and $\sigma \geq 0$, then the solution of (2.2) is such that $u \geq 0$.

The next result will be useful.

Lemma 2.2 (Comparison result). Let $\lambda<0$ and $u \in C^{2}(\Omega)$. If

$$
-u^{\prime \prime}(x)-\lambda u(x) \geq 0, \quad x \in \Omega, \quad u(0) \geq 0, \quad u(\pi) \geq 0,
$$

then $u(x) \geq 0, x \in \Omega$.

Proof. If $u(y)<0$ for some $y \in \Omega$, set $u\left(x_{m}\right)=\min \{u(x): x \in \Omega\}<0$. Hence, $u^{\prime}\left(x_{m}\right)=0$ and $u^{\prime \prime}\left(x_{m}\right) \geq 0$. On the other hand, $-u^{\prime \prime}\left(x_{m}\right) \geq \lambda u\left(x_{m}\right)>0$ which is a contradiction.

Now, consider the following nonlinear Dirichlet problem

$$
\begin{gathered}
-u^{\prime \prime}(x)=f(x, u(x)), \quad x \in \Omega, \\
u(0)=u(\pi)=0,
\end{gathered}
$$


where $f: \Omega \times \mathbb{R} \rightarrow \mathbb{R}$ is continuous.

We say that $\alpha \in C^{2}(\Omega)$ is a lower solution of (2.3) if

$$
-\alpha^{\prime \prime}(x) \leq f(x, \alpha(x)), \quad x \in \Omega ; \quad \alpha(0) \leq 0, \quad \alpha(\pi) \leq 0 .
$$

Analogously, $\beta \in C^{2}(\Omega)$ is an upper solution of (2.3) if

$$
-\beta^{\prime \prime}(x) \geq f(x, \beta(x)), \quad x \in \Omega ; \quad \beta(0) \geq 0, \quad \beta(\pi) \geq 0 .
$$

Theorem 2.1 (Upper and Lower Solution Method). Suppose that $\alpha, \beta \in C^{2}(\Omega)$ are lower and upper solutions for (2.3), respectively, such that

$$
\alpha(x) \leq \beta(x) \quad \text { for every } x \in \Omega .
$$

Then there exists at least one solution $u$ of (2.3) such that $\alpha(x) \leq u(x) \leq \beta(x)$ for every $x \in \Omega$.

Proof. We sketch the proof since it follows standard arguments (see, for instance, $[3,8])$. Consider the modified problem

$$
-u^{\prime \prime}(x)=\hat{f}(x, u(x)), \quad x \in \Omega, \quad u(0)=u(\pi)=0,
$$

where $\hat{f}(x, u)=f(x, p(x, u)), p(x, u)=\max \{\alpha(x), \min \{u, \beta(x)\}\}$. Any solution $u$ of the modified problem is such that $\alpha \leq u \leq \beta$ on $\Omega$. Hence, any solution of (2.7) is also a solution of (2.3). On the other hand, (2.7) is solvable since $\hat{f}$ is bounded. This completes the proof.

Theorem 2.2 (Monotone Iterative Technique). Suppose that $\alpha, \beta \in C^{2}(\Omega)$ are lower and upper solutions for (2.3), respectively, satisfying (2.6). Moreover, assume that there exists $M>0$ such that

$$
f(x, u)-f(x, v) \geq-M(u-v), \quad x \in \Omega, \alpha(x) \leq v \leq u \leq \beta(x) .
$$

Then, there exist monotone sequences $\left\{\alpha_{n}\right\}$ and $\left\{\beta_{n}\right\}$ with $\alpha_{0}=\alpha, \beta_{0}=\beta, \alpha_{n} \leq$ $\beta_{m}$ for every $n, m \in \mathbb{N}$, and $\lim _{n \rightarrow \infty} \alpha_{n}=r, \lim _{n \rightarrow \infty} \beta_{n}=\rho$ uniformly on $\Omega$. Here, $r$ and $\rho$ are the minimal and maximal solutions of (2.3) between $\alpha$ and $\beta$, that is, if $u$ is a solution of (2.3) such that $\alpha(x) \leq u(x) \leq \beta(x)$ for every $x \in \Omega$, then $r(x) \leq u(x) \leq \rho(x)$ for every $x \in \Omega$.

Proof. For any $\eta \in C(\Omega)$ with $\alpha \leq \eta \leq \beta$ on $\Omega$, consider the linear Dirichlet problem

$$
-u^{\prime \prime}(x)+M u(x)=f(x, \eta(x))+M \eta(x), \quad x \in \Omega, \quad u(0)=u(\pi)=0 .
$$

Denote by $u=A \eta$ the unique solution of this problem. Using the comparison result of Lemma 2.2 it is easy to show that $\alpha \leq A \eta \leq \beta$. Moreover, if $\eta_{1}, \eta_{2} \in C(\Omega)$ are such that $\alpha \leq \eta_{1} \leq \eta_{2} \leq \beta$ on $\Omega$, then $\alpha \leq A \eta_{1} \leq A \eta_{2} \leq \beta$.

Now, defining $\alpha_{0}=\alpha, \alpha_{n}=A \alpha_{n-1}, n \geq 1$, we have that $\left\{\alpha_{n}\right\} \uparrow r$ monotonically and uniformly on $\Omega$, and $r$ is the minimal solution of (2.3) between $\alpha$ and $\beta$. Analogously, setting $\beta_{0}=\beta, \beta_{n}=A \beta_{n-1}, n \geq 1$, we obtain a decreasing sequence $\left\{\beta_{n}\right\} \downarrow \rho$ monotonically and uniformly on $\Omega$, and $\rho$ is the maximal solution of (2.3) between $\alpha$ and $\beta$. 


\section{Quasilinearization method}

To develop the quasilinearization technique for the nonlinear Dirichlet problem (2.3), assume that $\alpha$ and $\beta$ are lower and upper solutions of (2.3) respectively and that (2.6) holds. Thus, let

$$
S=\{(x, u) \in \Omega \times \mathbb{R}: \alpha(x) \leq u \leq \beta(x)\},
$$

and consider the following conditions:

$$
\begin{gathered}
\frac{\partial f}{\partial u}(x, u), \frac{\partial^{2} f}{\partial u^{2}}(x, u) \quad \text { exist and are continuous for every }(x, u) \in S, \\
\frac{\partial f}{\partial u}(x, u)<1, \quad \text { for every }(x, u) \in S .
\end{gathered}
$$

Note that (3.1) implies that there exists $m>0$ such that

$$
\frac{\partial^{2} f}{\partial u^{2}}(x, u) \geq-2 m, \quad(x, u) \in S .
$$

Now, for $x \in \Omega$ and $u, v \in \mathbb{R}$ with $\alpha(x) \leq v \leq u \leq \beta(x)$, define

$$
g(x, u, v)=f(x, v)+\left[\frac{\partial f}{\partial u}(x, v)+2 m v\right](u-v)-m\left(u^{2}-v^{2}\right) .
$$

Note that $g(x, u, v) \leq f(x, u)$ and that $g(x, u, u)=f(x, u)$.

Theorem 3.1. Assume that $\alpha$ and $\beta$ are lower and upper solutions of (2.3) such that (2.6) is valid. Further, suppose that (3.1) and (3.2) hold. Then, there exists a monotone sequence $\left\{w_{n}\right\}$ which converges uniformly to a solution of (2.3) and the convergence is quadratic.

Proof. Set $w_{0}=\alpha$, and consider the Dirichlet problem

$$
-u^{\prime \prime}(x)=g\left(x, u(x), w_{0}(x)\right), \quad x \in \Omega, \quad u(0)=u(\pi)=0 .
$$

It is easy to see that $w_{0}$ is a lower solution for (3.5) and that $\beta$ is an upper solution for (3.5). Therefore, by using Theorem 2.1, we have that there exists a solution $w_{1}$ of (3.5) such that $w_{0} \leq w_{1} \leq \beta$ on $\Omega$.

Now, consider the Dirichlet problem

$$
-u^{\prime \prime}(x)=g\left(x, u(x), w_{1}(x)\right), \quad x \in \Omega, \quad u(0)=u(\pi)=0 .
$$

In view of the fact that $w_{0} \leq w_{1} \leq \beta$ we get using (3.4) that $w_{1}$ is a lower solution for (3.6) and that $\beta$ is an upper solution for (3.6). As before, we have that (3.6) has a solution $w_{2}$ such that $w_{1} \leq w_{2} \leq \beta$ on $\Omega$.

This process can be continued successively to obtain a monotone sequence $\left\{w_{n}\right\}$ satisfying

$$
w_{0} \leq w_{1} \leq w_{2} \leq \cdots \leq w_{n-1} \leq w_{n} \leq \beta,
$$

where $w_{n}$ is a solution of the Dirichlet problem

$$
-u^{\prime \prime}(x)=g\left(x, u(x), w_{n-1}(x)\right), \quad x \in \Omega, \quad u(0)=u(\pi)=0 .
$$

The sequence $\left\{w_{n}\right\}$ is monotone and, in consequence, it has a pointwise limit $w$. To show that $w$ is in fact a solution of (2.3), notice that $w_{n}$ is a solution of the following linear Dirichlet problem:

$$
-u^{\prime \prime}(x)=\sigma_{n}(x), \quad x \in \Omega, \quad u(0)=u(\pi)=0,
$$


with $\sigma_{n}(x)=g\left(x, w_{n}(x), w_{n-1}(x)\right), x \in \Omega$. It is clear that the sequence $\left\{\sigma_{n}\right\}$ is bounded in $C(\Omega)$ since $g$ is continuous on $S$ and, for $n=1,2, \ldots$, we have that $\alpha \leq w_{n} \leq \beta$. Also,

$$
\lim _{n \rightarrow \infty} \sigma_{n}(x)=f(x, w(x)), \quad x \in \Omega
$$

On the other hand,

$$
w_{n}(x)=\int_{0}^{\pi} G_{0}(x, y) \sigma_{n}(y) d y .
$$

This implies that $\left\{w_{n}\right\}$ is bounded in $C^{2}(\Omega)$, and hence $\left\{w_{n}\right\} \uparrow w$ uniformly on $\Omega$. In consequence, passing to the limit when $n \rightarrow \infty$, we get that

$$
w(x)=\int_{0}^{\pi} G_{0}(x, y) f(y, w(y)) d y, \quad x \in \Omega,
$$

that is, $w$ is a solution of $(2.3)$.

Finally, to show that $\left\{w_{n}\right\} \rightarrow w$ quadratically, define

$$
F(x, u)=f(x, u)+m u^{2}, \quad(x, u) \in S,
$$

where $m$ was chosen so that (3.3) holds, and select a constant $c>0$ such that

$$
0 \leq \frac{\partial^{2} F}{\partial u^{2}}(x, u) \leq c, \quad(x, u) \in S .
$$

Let $e_{n}=w-w_{n}, n=1,2, \ldots$ For $x \in \Omega$, we have that

$$
\begin{aligned}
-e_{n}^{\prime \prime}(x)= & f(x, w(w))-g\left(x, w_{n}(x), w_{n-1}(x)\right) \\
=F(x, w(x))-F\left(x, w_{n-1}(x)\right)-\frac{\partial F}{\partial u}\left(x, w_{n-1}(x)\right) & \\
& \cdot\left[w_{n}(x)-w_{n-1}(x)\right]-m\left[w^{2}(x)-w_{n-1}^{2}(x)\right] .
\end{aligned}
$$

Using the mean value theorem repeatedly we have that there exists $\xi=\xi(x)$ and $\zeta=\zeta(x), w_{n}(x) \leq \zeta \leq \xi \leq \beta(x)$, such that

$$
\begin{aligned}
-e_{n}^{\prime \prime}(x)= & \frac{\partial F}{\partial u}(x, \xi(x)) \cdot\left[w(x)-w_{n-1}(x)\right]-\frac{\partial F}{\partial u}\left(x, w_{n-1}(x)\right) \\
& \cdot\left[w_{n}(x)-w_{n-1}(x)\right]-m\left[w^{2}(x)-w_{n-1}^{2}(x)\right] \\
= & {\left[\frac{\partial F}{\partial u}(x, \xi(x))-\frac{\partial F}{\partial u}\left(x, w_{n-1}(x)\right)\right] \cdot\left[w(x)-w_{n-1}(x)\right] } \\
& +\left[\frac{\partial F}{\partial u}\left(x, w_{n-1}(x)\right)-m\left(w(x)+w_{n}(x)\right)\right] \cdot\left[w(x)-w_{n}(x)\right] \\
= & \frac{\partial^{2} F}{\partial u^{2}}(x, \zeta(x)) \cdot\left[\xi(x)-w_{n-1}(x)\right] \cdot\left[w(x)-w_{n-1}(x)\right] \\
& +\left[\frac{\partial F}{\partial u}\left(x, w_{n-1}(x)\right)-m\left(w(x)+w_{n}(x)\right)\right] \cdot\left[w(x)-w_{n}(x)\right] .
\end{aligned}
$$

In consequence, the error $e_{n}$ satisfies the Dirichlet problem

$$
-e_{n}^{\prime \prime}(x)-a_{n}(x) e_{n}(x)=c e_{n-1}^{2}(x)+b_{n}(x), \quad x \in \Omega, \quad e_{n}(0)=e_{n}(\pi)=0,
$$

where

$$
a_{n}(x)=\frac{\partial F}{\partial u}\left(x, w_{n-1}(x)\right)-m\left[w(x)+w_{n}(x)\right]
$$


and

$$
b_{n}(x)=\frac{\partial^{2} F}{\partial u^{2}}(x, \zeta(x)) \cdot\left[\xi(x)-w_{n-1}(x)\right] \cdot\left[w(x)-w_{n-1}(x)\right]-c e_{n-1}^{2}(x) \leq 0 .
$$

We have that

$$
\lim _{n \rightarrow \infty} a_{n}(x)=f(x, w(x)) \quad \text { uniformly on } x \in \Omega \text {. }
$$

Using (3.2), we can choose $\lambda<1$ and $n_{0} \in \mathbb{N}$ such that for $n \geq n_{0}$,

$$
a_{n}(x)<\lambda, \quad x \in \Omega .
$$

Thus,

$$
\begin{gathered}
-e_{n}^{\prime \prime}(x)-\lambda e_{n}(x)=\left[a_{n}(x)-\lambda\right] e_{n}(x)(x)+c e_{n-1}^{2}(x)+b_{n}(x), \quad x \in \Omega, \\
e_{n}(0)=e_{n}(\pi)=0,
\end{gathered}
$$

and $e_{n}$ satisfies the following relation:

$$
e_{n}(x)=\int_{0}^{\pi} G_{\lambda}(x, y)\left[\left(a_{n}(x)-\lambda\right) e_{n}(y)+c e_{n-1}^{2}(y)+b_{n}(y)\right] d y
$$

Therefore, for $x \in \Omega$ we have that

$$
0 \leq e_{n}(x) \leq c \int_{0}^{\pi} G_{\lambda}(x, y) e_{n-1}^{2}(y) d y,
$$

and there exists a constant $C>0$ such that for $n \geq n_{0}$ we have

$$
\left\|e_{n}\right\| \leq C\left\|e_{n-1}\right\|^{2} \text {. }
$$

The proof is thus complete.

\section{REFERENCES}

1. R. Bellman, Methods of Nonlinear Analysis, Vol. II. Academic Press, New York, 1973.

2. R. Bellman and R. Kalaba, Quasilinearization and Nonlinear Boundary Value Problems. American Elsevier, New York, 1965. MR 31:2828

3. G. S. Ladde, V. Lakshmikantham and A. S. Vatsala, Monotone Iterative Techniques for Nonlinear Differential Equations. Pitman, Boston, 1985. MR 88g:35006

4. V. Lakshmikantham, An Extension of the Method of Quasilinearization. J. Optimization Theory Appl. 82 (1994), 315-321. MR 95d:34021

5. V. Lakshmikantham and S. Malek, Generalized Quasilinearization. Nonlinear World 1 (1994), 59-63. MR 95d:34020

6. V. Lakshmikantham and J. J. Nieto, Generalized Quasilinearization for Nonlinear First Order Differential Equations. Nonlinear Times and Digest 2 (1995), 1-10. MR 96c:34025

7. V. Lakshmikantham, N. Shahzad and J. J. Nieto, Methods of Generalized Quasilinearization for Periodic Boundary Value Problems, Nonlinear Anal. 27 (1996), 143-151. CMP 96:12

8. J. J. Nieto and A. Cabada, A Generalized Upper and Lower Solution Method for Nonlinear Second Order Ordinary Differential Equations. J. Applied Math. Stochastic Anal. 5 (1992), 157-166. MR 94c:34033

9. N. Shahzad and S. Sivasundaram, Further Generalization of Quasilinearization Method for Boundary Value Problems, Nonlinear Times and Digest 2 (1995), 59-68. MR 96b:34013

10. N. Shahzad and A. S. Vatsala, Improved Generalized Quasilinearization Method for Second Order Boundary Value Problems. Dynam. Systems Appl. 4 (1995), 79-85. MR 95i:34018

Departamento de Análisis Matemático, Facultad de Matemáticas, Universidad de Santiago de Compostela, Spain

E-mail address: amnieto@usc.es 